\title{
Real-Time Virus Trapping and Fluorescent Imaging in Microfluidic Devices
}

$\mathbf{X X X X}$

Vol. 0, No. 0

\author{
Demir Akin, Haibo Li, and Rashid Bashir* \\ Laboratory of Integrated Biomedical Micro/Nanotechnology and Applications (LIBNA), \\ Birck Nanotechnology Center, School of Electrical and Computer Engineering, and \\ Department of Biomedical Engineering, Purdue University, \\ West Lafayette, Indiana 47907
}

Received November 5, 2003

\begin{abstract}
We have used dielectrophoretic filters within microfluidic biochips to capture from a flow and image virus particles in real-time. The verification of capture was performed by fluorescently labeling the particles using dual or triple labeling. These nonmechanical filters can be very valuable in the sample preparation, purification, and concentration of viral particles from a mixed sample. The described imaging methodology can be used for real-time imaging of nanometer scale virus particles for analysis of capture, detection, and characterization of these particles within micro and nanoscale sensors.
\end{abstract}

Electron microscopy is often used for the routine imaging of virus particles; however, this method requires the samples to be dried, fixed, coated, and laboriously prepared for imaging, which may also compromise sample structural integrity. Similarly, atomic force microscopy and X-ray crystallography-based virus visualization methods are also incompatible with use in real-time in micro and nanoscale biosensors and microfluidic lab-on-chip devices, thus, the utility of these methods in imaging of dynamic behaviors of nanoscale objects in biochips can be very limited.

In the current study, vaccinia virus ${ }^{1}$ was used as nanometer size biological object for the demonstration of successful labeling of the entire virion and different compartments of the viral particles with fluorescent dyes, and their real-time optical visualization during their capture in a microfluidic biochip with dielectrophoretic filters. The method involves use of high-quantum yield fluorophores, digital imaging with highly sensitive charge-coupled imaging devices (CCD), low magnification image acquisition, and biochip-based dielectrophoretic filters. ${ }^{2,3}$ The fluorescently labeled particles were captured for visualization by first flowing them in a microfluidic biochip and then trapping with a dielectrophortic filter built using interdigitated electrode arrays, as shown in Figure 1a. The dielectrophoretic filter was constructed by chemical etching a chamber of $12 \mu \mathrm{m}$ deep and $350 \mu \mathrm{m}$ wide into single-crystal silicon substrate using tetramethylammonium hydroxide (TMAH) ${ }^{4}$ After a thermal oxidation, a metal (Ti/ Pt) pattern of interdigitated electrode array (10 $\mu \mathrm{m}$ line with

\footnotetext{
* Corresponding author: E-mail: bashir@ecn.purdue.edu.
}

$8 \mu \mathrm{m}$ space) was sputtered and defined at the bottom of the chamber. Another plasma-enhanced oxide was deposited, and fluid inlet and outlet microtubes were inserted into the ports etched by deep reactive ion etch (DRIE) to the two ends of the chamber. The top of the chamber was covered with a glass slide by anodic bonding. In the experiment, virus sample solutions in distilled water (conductivity $1.5 \mu \mathrm{S} / \mathrm{cm}$ ) were injected into the chamber using a syringe pump (SP200i, World Precision Instruments Inc.,) and a $250 \mu \mathrm{L}$ gastight luer-lock syringe (ILS250TLL, World Precision Instruments Inc.). The flow rate was set to be at $0.1 \mu \mathrm{L} / \mathrm{min}$, and precautions were taken to avoid air bubbles. A sine wave excitation was used at $1 \mathrm{MHz}$ and $\sim 7 \mathrm{~V}_{\mathrm{pp}}$ (into $50 \Omega$ ) generated by an HP 33120A signal generator. The virus particles experienced positive dielectrophoresis in DI water and were collected mostly at the electrode edges, as shown in Figure 1, where the electric field gradient is the maximum. During the operation of the biochip, live fluorescent pictures were acquired using the setup described in the methods and shown in Figure 1a to assess the operation and progression of the dielectrophoretic trapping and separation of the viral particles. In real time, the images were collected with a computer running a video image capture and analysis software. The software allowed for digital zooming and various image enhancements.

The enveloped particles of vaccinia virus partcles, $\sim 230$ $\times 400 \mathrm{~nm}$, primarily contain proteins (90\%), lipids (5\%), and viral DNA (3\%). ${ }^{1}$ Lipid membranes and structural proteins of the viruses were labeled with lipophilic carbocya- 

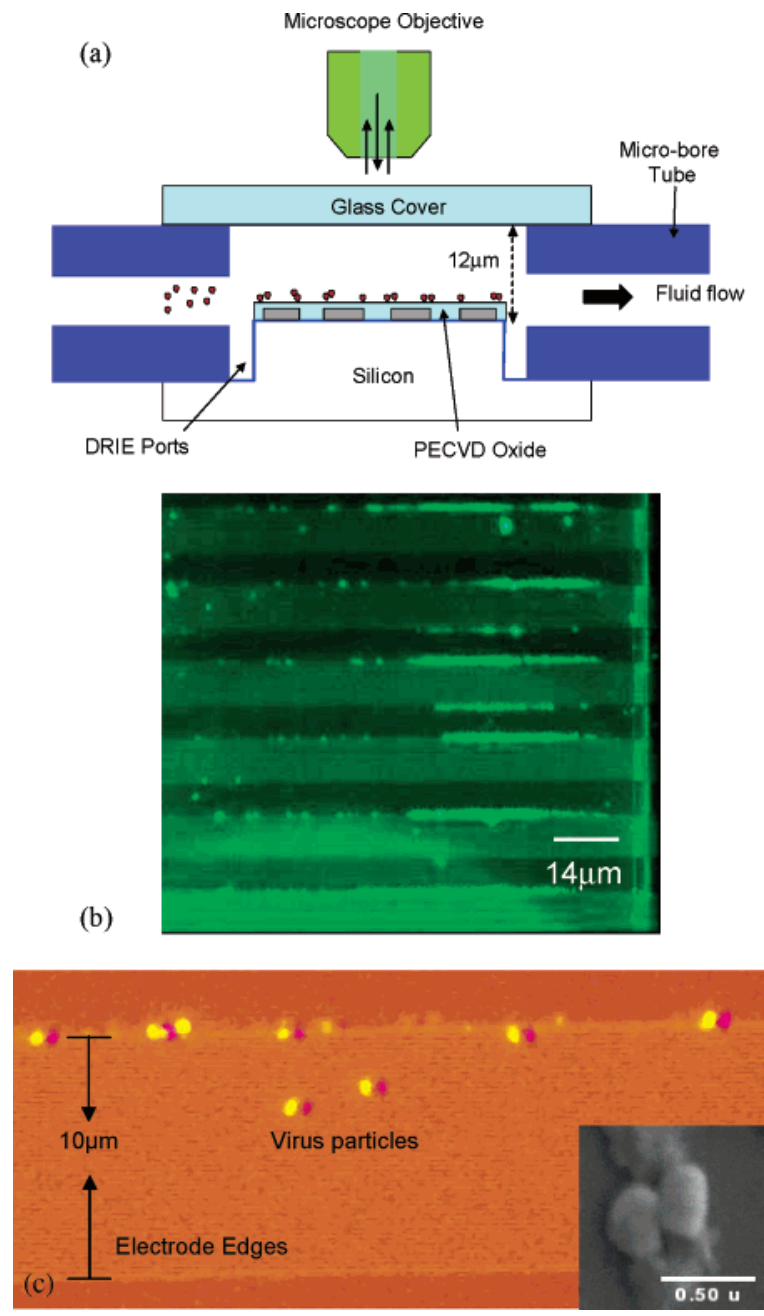

Figure 1. Dielectrophoretic capture and epifluorescence microscopic visualization of vaccinia virus particles. (a) Cross-sectional drawing of the microfluidic device, not drawn to scale. (b) Top view of fluorescently labeled western reserve strain of vaccinia virus particles trapped at the edges of the electrode inside a biochip and visualized through a FITC filter and a digital camera. Scale bar is $14 \mu \mathrm{m}$. (c) The dual $\left(\mathrm{DiOC}_{6} 3\right.$, green and DiL, red) labeled viral particles were trapped at the edges of the interdigitated DEP electrodes since their dielectric constant is greater than the medium. Scanning electron microscope (inset 1c) and fluorescent pictures of the virus particles correlate well morphologically. The scale bar is $0.5 \mu \mathrm{m}$ in the inset.

nine dyes $\mathrm{DiOC}_{6} 3$ and $\mathrm{DiL}$, and the viral nucleic acids were labeled with nucleophilic dyes Hoechst 33342 stain or YOYO1 (all of the stains used were from Molecular Probes, Eugene, OR). Western reserve strain of vaccinia virus was obtained as gradient purified virus. To stain the viral particles, the suspension containing virus particles was incubated with a mixture of either $\mathrm{DiOC}_{6} 3$ or DiL, $1 \mu \mathrm{M}$ final concentration, and either Hoechst dye or YOYO-1 ( $\mu \mathrm{M}$ final concentrations) and incubated at room temperature for $20 \mathrm{~min}$ after which they were subjected to $20,000 \mathrm{~g}$ centrifugation for $10 \mathrm{~min}$. The viral pellets were rinsed three times by resuspending in distilled water and centrifugation as above. Final virus pellets were resuspended in distilled water and kept at $4{ }^{\circ} \mathrm{C}$ until being used in the experiments. The fluorotracers used in this study often have 1000 -fold fluorescence enhancements upon

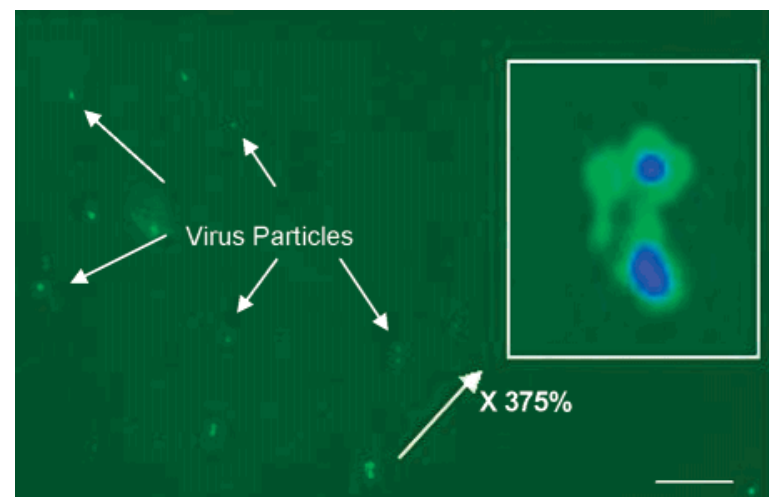

Figure 2. Labeling of internal viral DNA of vaccinia virus. Duallabeled viruses observed by digital epifluorescence microscopy at $400 \times$ magnification where the viral surface lipid membrane labeled green $\left(\mathrm{DiOC}_{6} 3\right)$ and viral nucleic acids were stained light blue (Hoechst 33342 stain) indicating penetration and intercalation of the nucleophilic dye. Separate green and blue channel images were acquired and digitally merged to reveal co-localization of the virus particles and viral DNAs. Further, 375\% digital zooming was performed to enhance the particle morphologies and staining.

binding to lipids and nucleic acids; furthermore, the nuclophilic dyes used have very high affinity for nucleic acids, with little or no staining of other biopolymers. Multicolor imaging was accomplished with a epifluorescent microscope and a digital CCD camera with up to $10 \times$ digital zoom (Pixera, Los Gatos, CA). Low magnification objectives $(20 \times$, $\mathrm{NA}=0.45$ and $40 \times, \mathrm{NA}=0.60)$ and filters appropriate for the dyes were used to collect separate images, which were merged, and contrast enhanced digitally using ImageJ program (http://rsb.info.nih.gov/ij/). The fluorophores were able to stain viral lipoproteins and the envelopes (green, $\mathrm{DiOC}_{6} 3$, Figure 1b,c and Figure 2; and red, DiL, Figure 1c). The method was applied to monitor performance of microfabricated dielectrophoretic filter chip designed to capture virus particle. Dual labeling of the virus particles was also successful since both nucleophilic dyes were able to penetrate through the $\mathrm{DiOC}_{6} 3$ labeled viral capsids and uniformly stain the viral nucleic acids blue (Hoechst 33342 stain, Figure 2). The viral morphology and physical dimensions of the virus particles from fluorescence images correlated well with the SEM images (Figure 1c and inset).

Optical resolution $(R)$ for an objective is given by $R=$ $\lambda /(2 \mathrm{NA})$, where $\lambda$ is wavelength and NA is numerical aperture. Note that the optical resolution is not affected by the magnification power of the objective used. The relationship between light intensity $(I)$ and magnification $(M)$ is given by $I \approx(\mathrm{NA})^{4}(M)^{-2}$; hence, higher magnification power occurs at the expense of acquired light intensity. More light is collected and available for digital enhancement by the low magnification objectives than that of high magnification. Consecutively, the loss from the optical magnification can be compensated through digital magnification. To further increase the usefulness of the method, multiple filter sets could be combined to obtain simultaneous emission spectrums from different fluorophores with differential affinity for different types of biological materials (lipophilic, nucleophilic, specific for carbohydrates, etc.), and one could 
greatly enhance co-localization and interactions of different components. Fluorescence-based labeling and low magnification optical imaging coupled with highly sensitive digital image acquisition open new possibilities for dynamic visualization of nanometer-sized biological entities and single molecules. Unlike high power magnification, the low magnification optical imaging approach limits the photobleaching and increases the fluorescence lifetime of the stained samples since the fluorophore molecules are exposed to lesser intensity light.

The method presented here allows for real-time observation of capture, detection, and intermolecular interaction events of viral particles within microfluidic biochips and other emerging applications such as verification of molecular transport and delivery using virus particles. The method may also be valuable for analysis of the interaction or co- localization of labeled nucleic acids with viral nucleocapsid and structural proteins by utilization of fluorophores with differential affinity for different types of biological materials.

Acknowledgment. We acknowledge the funding from the NIH-Bioengineering division (grant R21-EB000778-01). We also acknowledge Prof. S. Broyles for providing VV and Dr. D. Sherman for the SEM micrographs.

\section{References}

(1) Fields, B. N.; Knipe, D. M.; Howley, P. M. Virology; LippincottRaven Publishers: Philadelphia, 1996.

(2) Pohl, H. A. J. Appl. Phys. 1951, 22, 869-871.

(3) Markx, G. H.; Pethig, R. Biotechnol. Bioeng. 1995, 45, 337-343.

(4) Li, H., Akin, D., Bashir, R., submitted for publication.

NL034987P 